\title{
Asymptotic Behavior Analysis of a Fractional-Order Tumor-Immune Interaction Model with Immunotherapy
}

\author{
Wenbin Yang $\left(\mathbb{D},{ }^{1}\right.$ Xiaozhou Feng $\mathbb{D}^{2},{ }^{2}$ Shuhui Liang $\mathbb{D},{ }^{3}$ and Xiaojuan Wang $\mathbb{D}^{3}$ \\ ${ }^{1}$ School of Science, Xi'an University of Posts and Telecommunications, Xi'an, Shaanxi 710121, China \\ ${ }^{2}$ School of Science, Xi'an Technological University, Xi'an, Shaanxi 710032, China \\ ${ }^{3}$ Xijing Hospital of Digestive Diseases, Xijing Hospital, Air Force Medical University of PLA, Xi'an, Shaanxi 710032, China \\ Correspondence should be addressed to Xiaojuan Wang; wangxjfmmu@126.com
}

Received 23 February 2020; Revised 15 March 2020; Accepted 17 March 2020; Published 28 April 2020

Academic Editor: Marcelo Messias

Copyright (c) 2020 Wenbin Yang et al. This is an open access article distributed under the Creative Commons Attribution License, which permits unrestricted use, distribution, and reproduction in any medium, provided the original work is properly cited.

A fractional-order tumor-immune interaction model with immunotherapy is proposed and examined. The existence, uniqueness, and nonnegativity of the solutions are proved. The local and global asymptotic stability of some equilibrium points are investigated. In particular, we present the sufficient conditions for asymptotic stability of tumor-free equilibrium. Finally, numerical simulations are conducted to illustrate the analytical results. The results indicate that the fractional order has a stabilization effect, and it may help to control the tumor extinction.

\section{Introduction}

Tumor or tumour is a term used to describe the name for a swelling or lesion formed by an abnormal growth of cells. A tumor can be benign, premalignant, or malignant, whereas cancer is by definition malignant and is used to describe a disease in which abnormal cells divide without control and are able to invade other tissues. Cancer cells can spread to other parts of the body through blood and lymph systems [1], and so cancer is known as the leading cause of death in the world. During the last four decades, a large body of evidence has accumulated to provide support for the concept that the host immune system interacts with developing tumors and may be responsible for the arrest of tumor growth and for tumor regression [2].

Immunotherapy holds much promise for the treatment option and considered the fourth-line cancer therapy [3] by using cytokines and adoptive cellular immunotherapy (ACI) since adoptive immunotherapy using lymphokine-activated killer (LAK) cells or tumor-infiltrating lymphocytes (TIL) plus IL-2 has yielded positive results both in experimental tumor models and clinical trials [4].

The most current terminology used to describe cytokines is "immunomodulating agents" which are important regulators of both the innate and adaptive immune response. Examples of cytokines are protein hormones produced mainly by activated T cells (lymphocytes) in cellmediated immunity, and interleukin-2 (IL-2), produced mainly by $\mathrm{CD} 4^{+} \mathrm{T}$ cells, is the main cytokine responsible for lymphocyte activation, growth, and differentiation. ACI refers to the injection of cultured immune cells that have antitumor reactivity into the tumor-bearing host, which is typically achieved in conjunction with large amounts of IL-2 by using the following two methods: LAK therapy and TIL therapy. For more information on cytokines and ACI, the reader is referred to [5] and the references therein.

By applying each therapy separately or by applying both therapies simultaneously, Kirschner and Panetta [6] considered a model describing tumor-immune dynamics together with the feature of IL-2 dynamics. They proposed a 
model describing the interaction between the effector cells, tumor cells, and the cytokine (IL-2):

$$
\left\{\begin{array}{l}
\frac{\mathrm{d} E}{\mathrm{~d} t}=c T-\mu_{2} E+\frac{p_{1} E I_{L}}{g_{1}+I_{L}}+s_{1}, \\
\frac{\mathrm{d} T}{\mathrm{~d} t}=r_{2} T(1-b T)-\frac{a E T}{g_{2}+T}, \\
\frac{\mathrm{d} I_{L}}{\mathrm{~d} t}=\frac{p_{2} E T}{g_{3}+T}-\mu_{3} I_{L}+s_{2}, \\
E(0)=E_{0}, T(0)=T_{0}, I_{L}(0)=I_{L_{0}}
\end{array}\right.
$$

where $E(t)$ represents the activated immune system cells (commonly called effector cells) such as cytotoxic T cells, macrophages, and natural killer cells that are cytotoxic to the tumor cells; $T(t)$ represents the tumor cells; and $I_{L}(t)$ represents the concentration of IL-2 in the single tumor-site compartment. The parameters and their biological interpretations are summarized in Table 1.

For the nondimensionalized model (1), we adopt the following scaling:

$$
\begin{aligned}
& \widetilde{t}=r_{2} t, \\
& u=E \text {, } \\
& v=\frac{T}{g_{3}}, \\
& w=\frac{I_{L}}{g_{1}}, \\
& \tilde{b}=b_{g_{3}} \text {, } \\
& \tilde{c}=\frac{c g_{3}}{r_{2}}, \\
& \tilde{\mu}_{2}=\frac{\mu_{2}}{r_{2}}, \\
& \tilde{p}_{1}=\frac{p_{1}}{r_{2}}, \\
& \widetilde{s}_{1}=\frac{s_{1}}{r_{2}}, \\
& \widetilde{s}_{2}=\frac{s_{2}}{g_{1} r_{2}}, \\
& \tilde{\mu}_{3}=\frac{\mu_{3}}{r_{2}}, \\
& \tilde{a}=\frac{a}{g_{3} r_{2}}, \\
& \tilde{g}=\frac{g_{2}}{g_{3}}, \\
& \tilde{p}_{2}=\frac{p_{2}}{g_{1} r_{2}} \text {. }
\end{aligned}
$$

TABle 1: Parameters and their biological meanings.

\begin{tabular}{lc}
\hline Parameter & Biological meaning \\
\hline$c$ & Antigenicity of the tumor \\
$\left(1 / \mu_{2}\right)$ & Natural average lifespan \\
$p_{1}, a, p_{2}$ & Uptake velocity when all sites are saturated by the \\
& substrate \\
$s_{1}$ & Treatment by an external source of effector cells \\
$r_{2}$ & (ACI) \\
$(1 / b)$ & Net per capita growth rate \\
$g_{i}(i=1,2,3)$ & Environmental carrying capacity \\
$\mu_{3}$ & Half saturation constant \\
$s_{2}$ & Loss/degraded rate of IL-2 \\
\hline
\end{tabular}

Then model (1) is converted into the following form (dropping the tilde):

$$
\left\{\begin{array}{l}
\frac{\mathrm{d} u}{\mathrm{~d} t}=c v-\mu_{2} u+\frac{p_{1} u w}{1+w}+s_{1}, \\
\frac{\mathrm{d} v}{\mathrm{~d} t}=v(1-b v)-\frac{a u v}{g+v} \\
\frac{\mathrm{d} w}{\mathrm{~d} t}=\frac{p_{2} u v}{1+v}-\mu_{3} w+s_{2}, \\
u(0)=u_{0}, v(0)=v_{0}, w(0)=w_{0} .
\end{array}\right.
$$

In recent years, fractional-order differential equations have attracted the attention of researchers due to their ability to provide a good description of certain nonlinear phenomena. The fractional-order differential equations are generalizations of ordinary differential equations to arbitrary (noninteger) orders. Some researchers studied the fractional-order differential equations to describe complex systems in different branches of physics, chemistry, and engineering [7]. In the last few years, many researchers have also employed fractional-order biological models [8]. This is because fractional-order differential equations are naturally related to systems with memory [8]. Many biological systems possess memory, and the conception of the fractional-order system may be closer to real-life situations than integer-order systems. The advantages of fractional-order systems are that they describe the whole time domain for physical processes, while the integer-order model is related to the local properties of a certain position, and they allow greater degrees of freedom in the model [9]. The relevant works related to the fractional modeling can be found in [10-13] and the references therein.

To the best of the authors' knowledge, the dynamical analysis of a fractional-order tumor-immune interaction system with immunotherapy has not been performed before. Motivated by the above considerations, in this paper, we study a fractional-order tumor-immune interaction system by extending the integer order model (3) as follows: 


$$
\left\{\begin{array}{l}
{ }_{0}^{c} D_{t}^{\alpha} u(t)=c v-\mu_{2} u+\frac{p_{1} u w}{1+w}+s_{1}, \\
{ }_{0}^{c} D_{t}^{\alpha} v(t)=v(1-b v)-\frac{a u v}{g+v}, \\
{ }_{0}^{c} D_{t}^{\alpha} w(t)=\frac{p_{2} u v}{1+v}-\mu_{3} w+s_{2}, \\
u(0)=u_{0} \geq 0, v(0)=v_{0} \geq 0, w(0)=w_{0} \geq 0,
\end{array}\right.
$$

where $\alpha \in(0,1)$ and ${ }_{0}^{c} D_{t}^{\alpha}$ is the standard Caputo differentiation. The Caputo fractional derivative of order $\alpha$ is defined as $[9,14]$

$$
\begin{array}{r}
{ }_{t_{0}}^{c} D_{t}^{\alpha} f(t)=\frac{1}{\Gamma(n-\alpha)} \int_{t_{0}}^{t}(t-s)^{n-\alpha-1} f^{(n)}(s) \mathrm{d} s, \\
n-1<\alpha<n, n \in \mathbb{N} .
\end{array}
$$

In this paper, we consider immunotherapy to be $\mathrm{ACI}$ and/or IL-2 delivery either separately or in combination in the interaction site among effector cells, the tumor, and IL-2. The organization of this paper is as follows. In Section 2, the existence, uniqueness, and nonnegativity of the fractionalorder model (4) are presented. In Section 3, equilibria and (global) asymptotic stability analysis of the fractional-order model (4) are given. The numerical simulations are provided to verify the theoretical results of the fractional-order model (4) in Section 4. Finally, the study concludes with a brief discussion in Section 5.

\section{Existence, Uniqueness, and Nonnegativity}

This section studies the existence, uniqueness, and nonnegativity of the solutions of the fractional-order model (4).
To prove the existence and uniqueness of the solution for model (4), we need the following lemma.

Lemma 1 (see $[8,15])$. Consider the system

$$
{ }_{t_{0}}^{c} D_{t}^{\alpha} x(t)=f(t, x)
$$

with initial condition $x\left(t_{0}\right)$, where $\alpha \in(0,1)$ and $f:\left[t_{0}, \infty\right) \times \Omega \longrightarrow \mathbb{R}^{N}, \Omega \in \mathbb{R}^{N}$, if $f(t, x)$ satisfies the locally Lipschitz condition with respect to $x$, then there exists a unique solution of (6) on $\left[t_{0}, \infty\right) \times \Omega$.

Definition 1 (see [16]). A point $x^{*}$ is called an equilibrium point of system (6) if and only if $f\left(t, x^{*}\right)=0$.

Theorem 1. Let $\Omega=\left\{(u, v, w) \in \mathbb{R}^{3}: \max \{|u|,|v|,|w|\} \leq\right.$ $M\}$. For each initial condition $X_{0}=\left(u_{0}, v_{0}, w_{0}\right) \in \Omega$, there exists a unique solution of the fractional-order model (4), which is defined for all $t \geq 0$.

Proof. Let $0<T<\infty$. We seek a sufficient condition for existence and uniqueness of the solutions of the fractionalorder model (4) in the region $\Omega \times(0, T]$. We denote $X=(u, v, w)$ and $\bar{X}=(\bar{u}, \bar{v}, \bar{w})$. Consider a mapping $F(X)=\left(F_{1}(X), F_{2}(X), F_{3}(X)\right)$, where

$$
\begin{aligned}
& F_{1}(X)=c v-\mu_{2} u+\frac{p_{1} u w}{1+w}+s_{1}, \\
& F_{2}(X)=v(1-b v)-\frac{a u v}{g+v} \\
& F_{3}(X)=\frac{p_{2} u v}{1+v}-\mu_{3} w+s_{2} .
\end{aligned}
$$

For any $X, \bar{X} \in \Omega$, it follows from (4) that

$$
\begin{aligned}
\| & F(X)-F(\bar{X}) \| \\
= & \left|c v-\mu_{2} u+\frac{p_{1} u w}{1+w}+s_{1}-c \bar{v}+\mu_{2} \bar{u}-\frac{p_{1} \overline{u w}}{1+\bar{w}}-s_{1}\right|+\left|v(1-b v)-\frac{a u v}{g+v}-\bar{v}(1-b \bar{v})+\frac{a \bar{u} v}{g+\bar{v}}\right| \\
& +\left|\frac{p_{2} u v}{1+v}-\mu_{3} w+s_{2}-\frac{p_{2} \overline{u v}}{1+\bar{v}}+\mu_{3} \bar{w}-s_{2}\right|+\left|\left[1-b(v+\bar{v})-\frac{a g \bar{u}}{(g+u)(g+\bar{u})}\right](v-\bar{v})+\frac{a v}{g+v}(u-\bar{u})\right| \\
= & \left|c(v-\bar{v})+\left(\frac{p_{1} w}{1+w}-\mu_{2}\right)(u-\bar{u})+\frac{p_{1} \bar{u}}{(1+w)(1+\bar{w})}(w-\bar{w})\right|+\left|\frac{p_{2} v}{1+v}(u-\bar{u})+\frac{p_{2} \bar{u}}{(1+v)(1+\bar{v})}(v-\bar{v})-\mu_{3}(w-\bar{w})\right| \\
\leq & c|v-\bar{v}|+\left(p_{1}+\mu_{2}\right)|u-\bar{u}|+p_{1} M|w-\bar{w}|+\left(1+2 b M+\frac{a M}{g}\right)|v-\bar{v}|+a|u-\bar{u}|+p_{2}|u-\bar{u}|+p_{2} M|v-\bar{v}|+\mu_{3}|w-\bar{w}| \\
= & \left(p_{1}+\mu_{2}+a+p_{2}\right)|u-\bar{u}|+\left(c+1+2 b M+\frac{a M}{g}+p_{2} M\right)|v-\bar{v}|+\left(p_{1} M+\mu_{3}\right)|w-\bar{w}| \\
\leq & L\|X-\bar{X}\|,
\end{aligned}
$$


where $\quad L=\max \left\{p_{1}+\mu_{2}+a+p_{2}, c+1+2 b M+\right.$ $\left.(a M / g)+p_{2} M, p_{1} M+\mu_{3}\right\}$. Thus, $F(X)$ satisfies the Lipschitz condition with respect to $X$. Consequently, it follows from Lemma 1 that there exists a unique solution of model (4).

Theorem 2. Let $\mathbb{R}_{+}=\{x \in \mathbb{R}: x \geq 0\} \quad$ and $\Omega_{+}=\left\{(u, v, w) \in \Omega: u \in \mathbb{R}_{+}, v \in \mathbb{R}_{+}\right.$and $\left.w \in \mathbb{R}_{+}\right\}$. For each initial condition $X_{0}=\left(u_{0}, v_{0}, w_{0}\right) \in \mathbb{R}_{+}^{3}$, all the solutions of the fractional-order model (4) are nonnegative.

Proof. We will prove this theorem by contradiction. Suppose there exists $t_{*} \geq 0$ at which the solutions of model (4) passes through either the $u$-axis, $v$-axis, or $w$-axis. Let $\alpha \in(0,1)$, then there are three possibilities:

(1) Assume that $u\left(t_{*}\right)=0, v\left(t_{*}\right)>0$, and $w\left(t_{*}\right)>0$. Then, there exists $t^{*}>t_{*}$ with $\left|t^{*}-t_{*}\right| \ll 1$, such that $u(t)<0, v(t)>0$ and $w(t)>0$ when $t \in\left(t^{*}, t_{*}\right]$. By the first equation of model (4), we have ${ }_{0}^{c} D_{t}^{\alpha} u(/)>\left(p_{1}-\mu_{2}\right) u$ for all $t \in\left(t^{*}, t_{*}\right]$, and then $u(t)>u(0) E_{\alpha}\left(\left(p_{1}-\mu_{2}\right) t^{\alpha}\right)$ for all $t \in\left(t^{*}, t_{*}\right]$. Recall that $E_{\alpha, \beta}(t)=\sum_{k=0}^{\infty}\left(t^{k} / \Gamma(k \alpha+\beta)\right)$ is the MittagLeffler function with $\alpha, \beta>0$ [17] and $E_{\alpha}=E_{\alpha, 1}$. Using the standard comparison theorem for fractional order and the positivity of Mittag-Leffler function $E_{\alpha}(t)>0$ [17], for any $\alpha \in(0,1), u(t)>0$ for all $t \in\left(t^{*}, t_{*}\right]$, which is a contradiction.

(2) Assume that $v\left(t_{*}\right)=0, u\left(t_{*}\right)>0$, and $w\left(t_{*}\right)>0$. Then, there exists $t^{*}>t_{*}$ with $\left|t^{*}-t_{*}\right| \ll 1$, such that $u(t)>0, v(t)<0$, and $w(t)>0$ when $t \in\left(t^{*}, t_{*}\right]$. By the second equation of model (4), we have ${ }_{0}^{c} D_{t}^{\alpha} v(t)>v$ for all $t \in\left(t^{*}, t_{*}\right]$, and then $v(t)>v(0) E_{\alpha}\left(t^{\alpha}\right)$ for all $t \in\left(t^{*}, t_{*}\right]$. So, $v(t)>0$ for all $t \in\left(t^{*}, t_{*}\right]$, which is a contradiction.

(3) Assume that $w\left(t_{*}\right)=0, u\left(t_{*}\right)>0$, and $v\left(t_{*}\right)>0$. Then, there exists $t^{*}>t_{*}$ with $\left|t^{*}-t_{*}\right| \ll 1$, such that $u(t)>0, v(t)>0$ and $w(t)<0$ when $t \in\left(t^{*}, t_{*}\right]$. By the second equation of model (4), we have ${ }_{0}^{c} D_{t}^{\alpha} w(t)>-\mu_{3} w$ for all $t \in\left(t^{*}, t_{*}\right]$, and then $w(t)>w(0) E_{\alpha}\left(\left(-\mu_{3}\right) t^{\alpha}\right)$ for all $t \in\left(t^{*}, t_{*}\right]$. So, $w(t)>0$ for all $t \in\left(t^{*}, t_{*}\right]$, which is a contradiction.

Therefore, the solution of model (4) will be nonnegative.

\section{Equilibria Analysis and Asymptotic Stability}

We investigate all nonnegative constant equilibrium points to (4). First, according to Definition 1, model (4) has the following four nonnegative equilibrium points, which have at least one component zero:

(1) $E_{1}=(0,0,0)$, if $s_{1}=0$ and $s_{2}=0$;

(2) $E_{2}=\left(\left(s_{1} / \mu_{2}\right), 0,0\right)$, if $s_{1}>0$ and $s_{2}=0$;

(3) $E_{3}=\left(0,0,\left(s_{2} / \mu_{3}\right)\right), \quad$ if $\quad s_{1}=0, \quad s_{2}>0, \quad$ and $\left(p_{1} s_{2} /\left(\mu_{3}+s_{2}\right)\right) \neq \mu_{2}$

(4) $E_{4}=\left(\left(s_{1}\left(\mu_{3}+s_{2}\right) /\left(\mu_{2} \mu_{3}+s_{2}\left(\mu_{2}-p_{1}\right)\right)\right), 0, I\right.$ $\left.\left(s_{2} / \mu_{3}\right)\right)$, if $s_{1}>0, s_{2}>0$, and $\mu_{2} \mu_{3}+s_{2}\left(\mu_{2}-p_{1}\right)>0$.
The cases (2) and (4) are realistic tumor-free equilibrium points. On the other hand, (1) and (3) are not realistic because the effector (or immune) cells do not disappear although the immune system can be weak. Thus, in this section, to investigate the tumor-free equilibrium points in (1), we examine the asymptotically stable behavior at the equilibrium points provided in the cases (2) and (4).

Next, we only provide the sufficient conditions of the existence of a unique positive equilibrium point $E_{*}=\left(u_{*}, v_{*}, w_{*}\right)$ to (4) and omit the proof process.

Lemma 2 (Lemma 2.1, see [18]). If one of the following inequalities

(1) $g c \geq(1-b g) s_{1},\left(\left(\mu_{2}-p_{1}\right) / a\right) g>s_{1}$ and $\mu_{2}>p_{1}$,

(2) $g c>s_{1},\left(\mu_{2} g / a\right)>s_{1}\left(\left(s_{2} / \mu_{3}\right)+1\right)$ and $\mu_{2}=p_{1}$,

holds, then (4) has a unique positive equilibrium point $E_{*}$.

Lemma 3. Let $J\left(x^{*}\right)$ denote the Jacobian matrix of system (6) evaluated at equilibrium point $x^{*}$. The eigenvalues of $J\left(x^{*}\right)$ are $\lambda_{i}$, where $i=1, \ldots, n$. Then, equilibrium point $x^{*}$ is locally asymptotically stable if and only if all eigenvalues $\lambda_{i}, i=$ $1, \ldots, n$ of $J\left(x^{*}\right)$ satisfy $\left|\arg \left(\lambda_{i}\right)\right|>(\alpha \pi / 2)$; equilibrium point $x^{*}$ is a saddle point if some eigenvalues $\lambda_{i}$ satisfy $\left|\arg \left(\lambda_{i}\right)\right|>(\alpha \pi / 2)$ and some others satisfy $\left|\arg \left(\lambda_{i}\right)\right|>(\alpha \pi / 2)$.

Now, we determine the local stability of the equilibrium points of model (4) using the linearization method. The Jacobian matrix of the system evaluated at point $X=(u, v, w)$ is given by

$J(X) \doteq F_{X}(X)=\left[\begin{array}{ccc}-\mu_{2}+\frac{p_{1} w}{1+w} & c & \frac{p_{1} u}{(1+w)^{2}} \\ -\frac{a v}{g+v} & 1-2 b v-\frac{a g u}{(g+v)^{2}} & 0 \\ \frac{p_{2} v}{1+v} & \frac{p_{2} u}{(1+v)^{2}} & -\mu_{3}\end{array}\right]$,

where $F(X)$ is defined in the proof of Theorem 1 .

Theorem 3. Equilibrium point $E_{2}=\left(\left(s_{1} / \mu_{2}\right), 0,0\right)$ of (4) is locally asymptotically stable if $a s_{1}>g \mu_{2}$ and is unstable, which is a saddle point, if $a s_{1}<g \mu_{2}$.

Proof. By (9), the Jacobian matrix of model (4) evaluated at equilibrium point $E_{2}=\left(\left(s_{1} / \mu_{2}\right), 0,0\right)$ is given by

$$
J\left(E_{2}\right)=\left[\begin{array}{ccc}
-\mu_{2} & c & \frac{p_{1} s_{1}}{\mu_{2}} \\
0 & 1-\frac{a s_{1}}{g \mu_{2}} & 0 \\
0 & \frac{p_{2} s_{1}}{\mu_{2}} & -\mu_{3}
\end{array}\right]
$$


Hence, the eigenvalues of $J\left(E_{2}\right)$ are $\lambda_{1}=-\mu_{2}, \lambda_{2}=-\mu_{3}$, and $\lambda_{3}=1-\left(a s_{1} / g \mu_{2}\right)$. Consequently, $\arg \left(\lambda_{1}\right)=\arg \left(\lambda_{2}\right)=$ $\pi$ and $\arg \left(\lambda_{3}\right)=\pi$ if $a s_{1}>g \mu_{2}$, which leads to $\left|\arg \left(\lambda_{i}\right)\right|>(\alpha \pi / 2), i=1,2,3, \quad$ for $0<\alpha<1$. Therefore, according to Lemma 3 , the equilibrium point $E_{2}$ is locally asymptotically stable.

If $a s_{1}<g \mu_{2}$, then $\arg \left(\lambda_{3}\right)=0$. Thus, $\left|\arg \left(\lambda_{3}\right)\right|<(\alpha \pi / 2)$ for $0<\alpha<1$, which yields that the equilibrium point $E_{2}$ is unstable and is a saddle point.
Theorem 4. Equilibrium point $E_{4}=\left(\left(s_{1}\left(\mu_{3}+s_{2}\right) /\left(\mu_{2} \mu_{3}+\right.\right.\right.$ $\left.\left.\left.s_{2}\left(\mu_{2}-p_{1}\right)\right)\right), 0, I\left(s_{2} / \mu_{3}\right)\right)$ of (4) is locally asymptotically stable if $p_{1} s_{2}>\mu_{2}\left(s_{2}+\mu_{3}\right)$ and $a s_{1}\left(s_{2}+\mu_{3}\right)>g\left[\mu_{2} \mu_{3}+s_{2}\left(\mu_{2}-p_{1}\right)\right]$; equilibrium point $E_{4}$ is a saddle point, if $p_{1} s_{2}<\mu_{2}\left(s_{2}+\mu_{3}\right)$ or $a s_{1}\left(s_{2}+\mu_{3}\right)<g\left[\mu_{2} \mu_{3}+s_{2}\left(\mu_{2}-p_{1}\right)\right]$.

Proof. By (9), the Jacobian matrix of model (4) evaluated at equilibrium point $E_{4}=\left(\left(s_{1} / \mu_{2}\right), 0,0\right)$ is given by

$$
J\left(E_{2}\right)=\left[\begin{array}{ccc}
-\mu_{2}+\frac{p_{1} s_{2}}{s_{2}+\mu_{3}} & c & \frac{p_{1}}{\left(1+s_{2} / \mu_{3}\right)^{2}} \cdot \frac{s_{1}\left(\mu_{3}+s_{2}\right)}{\mu_{2} \mu_{3}+s_{2}\left(\mu_{2}-p_{1}\right)} \\
0 & 1-\frac{a}{g} \cdot \frac{s_{1}\left(\mu_{3}+s_{2}\right)}{\mu_{2} \mu_{3}+s_{2}\left(\mu_{2}-p_{1}\right)} & 0 \\
0 & p_{2} \cdot \frac{s_{1}\left(\mu_{3}+s_{2}\right)}{\mu_{2} \mu_{3}+s_{2}\left(\mu_{2}-p_{1}\right)} & -\mu_{3}
\end{array}\right] \text {. }
$$

Hence, the eigenvalues of $J\left(E_{4}\right)$ are $\lambda_{1}=-\mu_{3}$, $\lambda_{2}=-\mu_{2}+\left(p_{10} s_{2} /\left(s_{2}+\mu_{3}\right)\right)$, and $\lambda_{3}=1-(a / g) \cdot\left(s_{1}\left(\mu_{3}+\right.\right.$ $\left.\left.s_{2}\right) /\left(\mu_{2} \mu_{3}+s_{2}\left(\mu_{2}-p_{1}\right)\right)\right)$. Consequently, $\arg \left(\lambda_{1}\right)=\pi$ and $\arg \left(\lambda_{2}\right)=\arg \left(\lambda_{3}\right)=\pi$ if $p_{1} s_{2}>\mu_{2}\left(s_{2}+\mu_{3}\right)$ and $a s_{1}\left(s_{2}+\right.$ $\left.\mu_{3}\right)>g\left[\mu_{2} \mu_{3}+s_{2}\left(\mu_{2}-p_{1}\right)\right]$, which leads to $\left|\arg \left(\lambda_{i}\right)\right|>(\alpha \pi / 2), i=1,2,3$, for $0<\alpha<1$. Therefore, according to Lemma 3 , the equilibrium point $E_{4}$ is locally asymptotically stable.

If $p_{1} s_{2}<\mu_{2}\left(s_{2}+\mu_{3}\right) \quad$ or $a s_{1}\left(s_{2}+\mu_{3}\right)>g\left[\mu_{2} \mu_{3}+s_{2}\right.$ $\left.\left(\mu_{2}-p_{1}\right)\right]$, then $\arg \left(\lambda_{2}\right)=0$ or $\arg \left(\lambda_{3}\right)=0$. Thus, $\left|\arg \left(\lambda_{2}\right)\right|$ or $\left|\arg \left(\lambda_{3}\right)\right|<(\alpha \pi / 2)$ for $0<\alpha<1$, which yields that the equilibrium point $E_{2}$ is a saddle point.

Remark 1. Note that equilibrium points $E_{1}$ and $E_{3}$ are not of biological significance since the effector (or immune) cells do not disappear although the immune system can be weak. As a supplement, we point out a fact that $E_{1}$ and $E_{3}$ are saddle in mathematics since the Jacobian matrix of model (4) evaluated at equilibrium points $E_{1}$ and $E_{3}$ are as follows:

$$
\begin{aligned}
& J\left(E_{1}\right)=\left[\begin{array}{ccc}
-\mu_{2} & c & 0 \\
0 & 1 & 0 \\
0 & 0 & -\mu_{3}
\end{array}\right], \\
& J\left(E_{3}\right)=\left[\begin{array}{ccc}
-\mu_{2}+\frac{p_{1} s_{2}}{s_{2}+\mu_{3}} & c & 0 \\
0 & 1 & 0 \\
0 & 0 & -\mu_{3}
\end{array}\right] .
\end{aligned}
$$

In what follows, the local stability of the unique interior equilibriums $E_{*}$ is investigated. By (9), the Jacobian matrix of model (4) evaluated at equilibrium point $E_{*}=\left(u_{*}, v_{*}, w_{*}\right)$ is given by

$$
J\left(E_{*}\right)=\left[\begin{array}{ccc}
-\mu_{2}+\frac{p_{1} w_{*}}{1+w_{*}} & c & \frac{p_{1} u_{*}}{\left(1+w_{*}\right)^{2}} \\
-\frac{a v_{*}}{g+v_{*}} & v_{*}\left[-b+\frac{a u_{*}}{\left(g+v_{*}\right)^{2}}\right] & 0 \\
\frac{p_{2} v_{*}}{1+v_{*}} & \frac{p_{2} u_{*}}{\left(1+v_{*}\right)^{2}} & -\mu_{3}
\end{array}\right] .
$$


The eigenvalues of Jacobian matrix $J\left(E_{*}\right)$ are the roots of where the following equation:

$$
P(\lambda)=\lambda^{3}+a_{1} \lambda^{2}+a_{2} \lambda+a_{3}=0,
$$

$$
\begin{aligned}
& a_{1}=\left(\mu_{2}-\frac{p_{1} w_{*}}{1+w_{*}}\right)+v_{*}\left[b-\frac{a u_{*}}{\left(g+v^{*}\right)^{2}}\right]+\mu_{3}, \\
& a_{2}=v_{*}\left(\mu_{2}-\frac{p_{1} w_{*}}{1+w_{*}}\right)\left[b-\frac{a u_{*}}{\left(g+v^{*}\right)^{2}}\right]+\mu_{3} v_{*}\left[b-\frac{a u_{*}}{\left(g+v^{*}\right)^{2}}\right]+\frac{a c v_{*}}{g+v^{*}}+\left[\mu_{3}\left(\mu_{2}-\frac{p_{1} w_{*}}{1+w_{*}}\right)-\frac{p_{1} u_{*}}{\left(1+w_{*}\right)^{2}} \frac{p_{2} v_{*}}{1+v_{*}}\right], \\
& a_{3}=v_{*}\left[b-\frac{a u_{*}}{\left(g+v^{*}\right)^{2}}\right]\left[\mu_{3}\left(\mu_{2}-\frac{p_{1} w_{*}}{1+w_{*}}\right)-\frac{p_{1} u_{*}}{\left(1+w_{*}\right)^{2}} \frac{p_{2} v_{*}}{1+v_{*}}\right]+\frac{p_{1} u_{*}}{\left(1+w_{*}\right)^{2}} \frac{p_{2} u_{*}}{\left(1+v_{*}\right)^{2}} \frac{a v_{*}}{g+v_{*}}+\mu_{3} \frac{a c v_{*}}{g+v_{*}} .
\end{aligned}
$$

The discriminant of $D(P)$ (see [19], Definition 1) is

$$
D(P)=18 a_{1} a_{2} a_{3}+a_{1}^{2} a_{2}^{2}-4 a_{1}^{3} a_{3}-4 a_{2}^{3}-27 a_{3}^{2} \text {. }
$$

By the Routh-Hurwitz conditions for fractional-order differential equations defined in [19], Proposition 1, we obtain the following results.

Theorem 5 (1) If $D(P)>0$ and $a_{1}>0, a_{3}>0, a_{1} a_{2}>a_{3}$, then $E_{*}$ is locally asymptotically stable for $\alpha \in(0,1)$.

(2) If $D(P)<0$ and $a_{1} \geq 0, a_{2} \geq 0, a_{3}>0$, then $E_{*}$ is locally asymptotically stable for $\alpha \in(0.5,(2 / 3))$.

(3) If $D(P)<0$ and $a_{1}<0, a_{2}<0$, then $E_{*}$ is unstable for $\alpha>(2 / 3)$.

(4) If $D(P)<0$ and $a_{1}>0, a_{2}>0, a_{1} a_{2}=a_{3}$, then $E_{*}$ is locally asymptotically stable for $\alpha \in(0,1)$.

Remark 2. It follows from Lemmas 2.2 and 2.3 in [18] that $\mu_{3}\left(\mu_{2}-\left(p_{1} w_{*} /\left(1+w_{*}\right)\right)\right)>\left(p_{1} u_{*} /\left(1+w_{*}\right)^{2}\right)\left(p_{2} v_{*} /(1+\right.$ $\left.\left.v_{*}\right)\right)$ and $\left(a c v_{*} /\left(g+v^{*}\right)\right)+v_{*}\left(\mu_{2}-\left(p_{1} w_{*} /\left(1+w_{*}\right)\right)\right)$ $\left[b-\left(a u_{*} /\left(g+v^{*}\right)^{2}\right)\right]>0$. So, the signs of some terms of $a_{i}, i=1,2,3$, could be determined.

We next investigate the global stability of the positive equilibrium point $E_{*}$ by introducing the following Lyapunov function:

$$
\begin{aligned}
E(t) \doteq & E(u(t), v(t), w(t))=\frac{1}{2}\left(u-u_{*}\right)^{2}+\left(v-v_{*}-v_{*} \ln \frac{v}{v_{*}}\right) \\
& +\frac{1}{2}\left(w-w_{*}\right)^{2},
\end{aligned}
$$

for the solution $(u, v, w)$ to (4). Note that $E(t) \geq 0$ for all $t \geq 0$, and thus, if ${ }_{0}^{c} D_{t}^{\alpha} E(t) \leq 0$ can be derived, then we obtain the desired result from the well-known Lyapunov stability.

Lemma 4 (see [20]). Let $x(t) \in \mathbb{R}_{+}$be a continuous and derivable function. Then, for any time instant $t>t_{0}$,

$$
{ }_{t_{0}}^{c} D_{t}^{\alpha}\left[x(t)-x^{*}-x^{*} \ln \frac{x(t)}{x^{*}}\right] \leq\left[1-\frac{x^{*}}{x(t)}\right]{ }_{t_{0}}^{c} D_{t}^{\alpha} x(t),
$$

where $x^{*} \in \mathbb{R}_{+}$and $\alpha \in(0,1)$.

Lemma 5 (see $[21,22])$. Let $\alpha \in(0,1), x \in C^{0}([0, T]: \mathbb{R})$, and $x^{\prime} \in L^{1}(0, T: \mathbb{R})$. Then,

$$
{ }_{t_{0}}^{c} D_{t}^{\alpha} x^{2}(t) \leq 2 x(t)_{t_{0}}^{c} D_{t}^{\alpha} x(t), \quad t \in(0, T] .
$$

Theorem 6. Assume that $g c \geq(1-b g) s_{1}$ and $\left(\left(\mu_{2}-p_{1}\right) / a\right) g>s_{1}$ where $\mu_{2}>p_{1}$. Then, the positive equilibrium point $E_{*}$ to (4) is globally asymptotically stable if

$$
\left\{\begin{array}{l}
\mu_{2} \geq p_{1}+\frac{c+(a / g)}{2}+\frac{1}{2}\left[p_{2}+p_{1} \frac{(c / b)+s_{1}}{\mu_{2}-p_{1}}\right], \\
b \geq \frac{1}{g}+\frac{c+(a / g)}{2}+\frac{p_{2}}{c} \frac{(c / b)+s_{1}}{\mu_{2}-p_{1}} \\
\mu_{3} \geq \frac{1}{2}\left[p_{2}+p_{1} \frac{(c / b)+s_{1}}{\mu_{2}-p_{1}}\right]+\frac{p_{2}}{2} \frac{(c / b)+s_{1}}{\mu_{2}-p_{1}}
\end{array}\right.
$$

Proof. Calculating the $\alpha$-order derivative of $E(t)$ along the solution of model (4), it follows Lemmas 4 and 5 that 


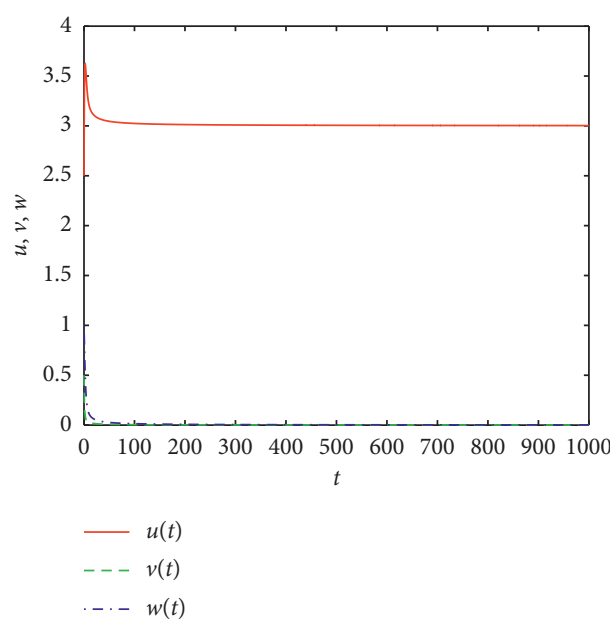

(a)

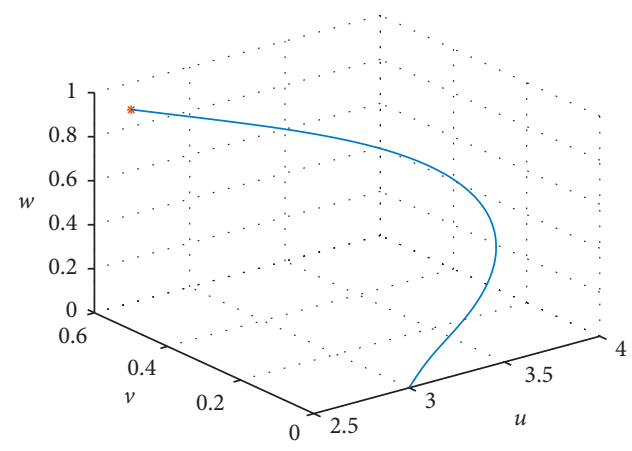

(b)

Figure 1: Time series (a) and phase diagram (b) of the fractional-order model (4) with $c=0.9, \mu_{2}=1, p_{1}=0.5, s_{1}=3, b=3, a=1, g=2.5, p_{2}=1, \mu_{3}=1, \alpha=0.9$, and $s_{2}=0$.

$$
\begin{aligned}
{ }_{0}^{c} D_{t}^{\alpha} E(t) & \leq\left(u-u_{*}\right)_{0}^{c} D_{t}^{\alpha} u(t)+\left[1-\frac{v^{*}}{v(t)}\right]{ }_{0}^{c} D_{t}^{\alpha} v(t)+\left(w-w_{*}\right)_{0}^{c} D_{t}^{\alpha} w(t) \\
& =\left(u-u_{*}\right)\left(c v-\mu_{2} u+\frac{p_{1} u w}{1+w}+s_{1}\right)+\left(v-v^{*}\right)\left(1-b v-\frac{a u}{g+v}\right)+\left(w-w_{*}\right)\left(\frac{p_{2} u v}{1+v}-\mu_{3} w+s_{2}\right) .
\end{aligned}
$$

By the definition of $E_{*}=\left(u_{*}, v_{*}, w_{*}\right)$, which is a coexistence equilibrium point of model (4), we have

$$
\begin{aligned}
{ }_{0}^{c} D_{t}^{\alpha} E(t) \leq & \left(u-u_{*}\right)^{2}\left\{-\mu_{2}+p_{1}+\frac{c+(a / g)}{2}+\frac{1}{2}\left[p_{2}+p_{1} \frac{c / b+s_{1}}{\mu_{2}-p_{1}}\right]\right\} \\
& +\left(v-v^{*}\right)^{2}\left(-b+\frac{1}{g}+\frac{c+(a / g)}{2}+\frac{p_{2}}{c} \frac{(c / b)+s_{1}}{\mu_{2}-p_{1}}\right) \\
& +\left(w-w_{*}\right)^{2}\left\{-\mu_{3}+\frac{1}{2}\left[p_{2}+p_{1} \frac{(c / b)+s_{1}}{\mu_{2}-p_{1}}\right]+\frac{p_{2}}{2} \frac{(c / b)+s_{1}}{\mu_{2}-p_{1}}\right\} .
\end{aligned}
$$

The conditions given in (20) guarantee that ${ }_{0}^{c} D_{t}^{\alpha} E(t) \leq 0$ for all $(u, v, w) \in \mathbb{R}_{+}^{3}$, and ${ }_{0}^{c} D_{t}^{\alpha} E(t)=0$ implies that $(u, v, w)=\left(u_{*}, v_{*}, w_{*}\right)$.

\section{Numerical Simulation}

In this section, numerical simulations of the fractional-order tumor-immune interaction model (4) are conducted to illustrate the theoretical results obtained before. The predictor-corrector PECE method of Adams-Bashforth-Moulton type [23] and some implicit fractional linear multistep methods (FLMMs) of the second order [24] are applied in order to find an approximate solution for our fractional-order model.
First, we choose the following set of parameters:

$$
\begin{aligned}
c & =0.9, \\
\mu_{2} & =1, \\
p_{1} & =0.5, \\
b & =3, \\
a & =1, \\
g & =2.5, \\
p_{2} & =1, \\
\mu_{3} & =1, \\
\alpha & =0.9,
\end{aligned}
$$




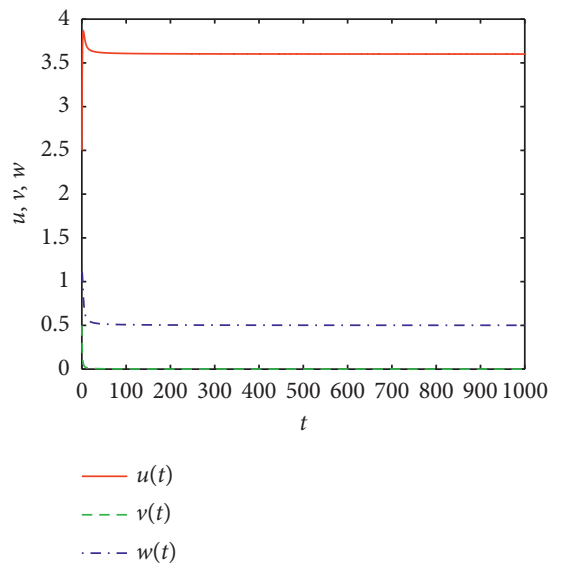

(a)

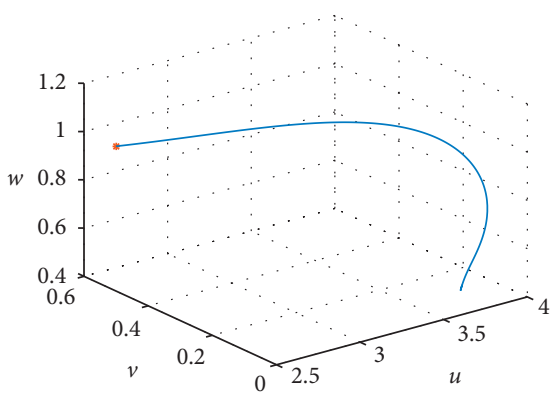

(b)

Figure 2: Time series (a) and phase diagram (b) of the fractional-order model (4) with $c=0.9, \mu_{2}=1, p_{1}=0.5, s_{1}=$ $3, b=3, a=1, g=2.5, p_{2}=1, \mu_{3}=1, \alpha=0.9$, and $s_{2}=0.5$.
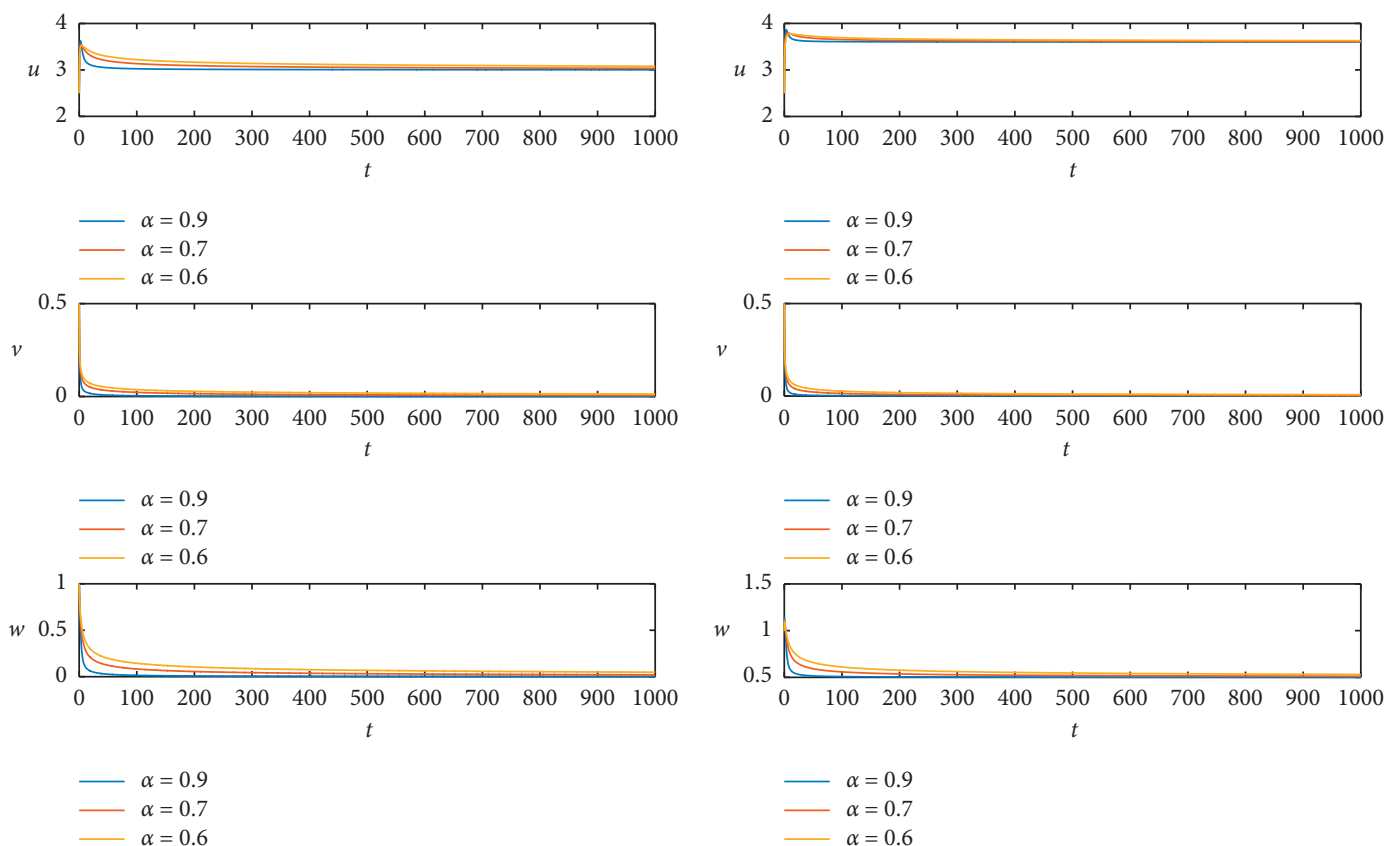

(a)

(b)

Figure 3: Time series of the fractional-order model (4) with $c=0.9, \mu_{2}=1, p_{1}=0.5, s_{1}=3, b=3, a=1, g=2.5, p_{2}=1, \mu_{3}=1$, and different values of the order $\alpha$ of the Caputo fractional derivative. (a) $s_{2}=0$ and (b) $s_{2}=0.5$.

and consider the asymptotic stability of the realistic tumor-free equilibria $E_{2}$ and $E_{4}$. This yields that under some conditions, the tumor can be cured thoroughly, by the therapy (ACI or ACI plus IL-2). Following Theorem 3, when $s_{1}=3$ and $s_{2}=0$, the realistic tumor-free equilibrium $E_{2}=\left(\left(s_{1} / \mu_{2}\right), 0,0\right)=(3,0,0)$ of the fractional-order model (4) is locally asymptotically stable as indicated in Figure 1.

Following Theorem 4, when $s_{1}=3$ and $s_{2}=0.5$, the realistic tumor-free equilibrium $E_{4}=\left(\left(s_{1}\left(\mu_{3}+s_{2}\right) /\left(\mu_{2} \mu_{3}+\right.\right.\right.$ $\left.\left.\left.s_{2}\left(\mu_{2}-p_{1}\right)\right)\right), 0, I\left(s_{2} / \mu_{3}\right)\right)=(3.6,0,0.5)$ of the fractionalorder model (4) is locally asymptotically stable as indicated in Figure 2.

For better visualization of the impact of $\alpha$ on the asymptotic rate of convergence of the realistic tumor-free equilibria $E_{2}$ and $E_{4}$, Figure 3 indicates that with the higher value of $\alpha$, the asymptotic rate of convergence of $E_{2}$ and $E_{4}$ will be larger.

Note that $v$ represents the tumor cells and $s_{1}$ and $s_{2}$ represent the treatment by an external source of effector 


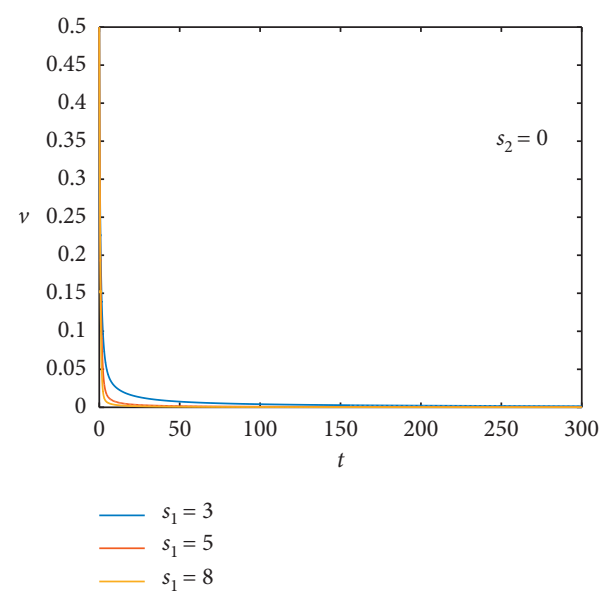

(a)

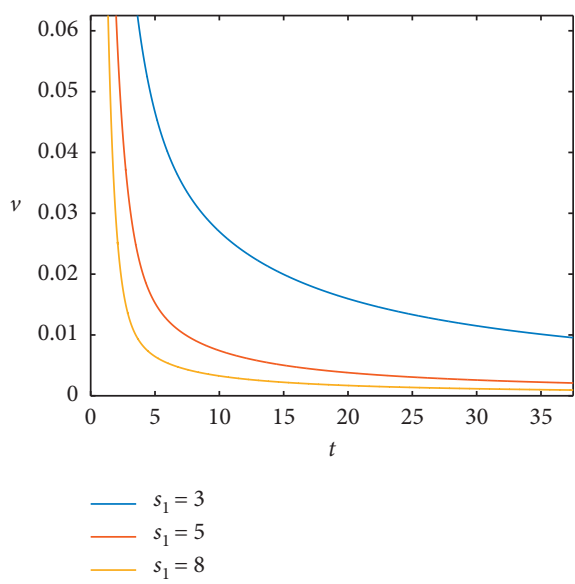

(b)

Figure 4: Time series of the immune system cells $v$ in the fractional-order model (4) with $c=0.9, \mu_{2}=1, p_{1}=0.5, b=3$, $a=1, g=2.5, p_{2}=1, \mu_{3}=1, s_{2}=0, \alpha=0.9$, and different treatment by an external input of IL-2 into the system $s_{1}$ : (a) original drawing and (b) drawing of partial enlargement of 4(a).

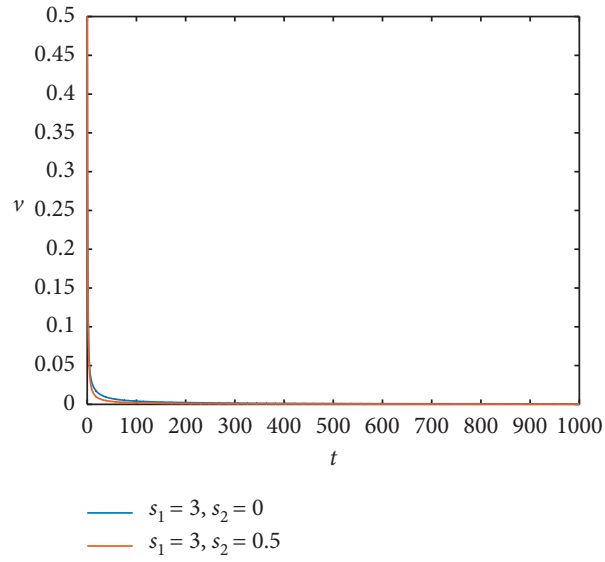

(a)

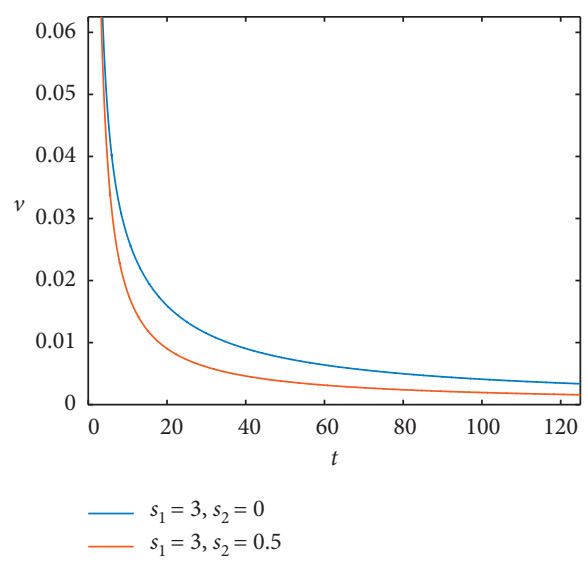

(b)

Figure 5: Time series of the immune system cells $v$ in the fractional-order model (4) with $c=0.9, \mu_{2}=1, p_{1}=0.5, s_{1}=3, b=3$, $a=1, g=2.5, p_{2}=1, \mu_{3}=1, \alpha=0.9$, and the same treatment by an external input of IL-2 into the system $s_{1}=3$. (a) Original drawing (the blue line represents that there is only the treatment by an external input of IL-2 into the system, i.e., $s_{2}=0$, and the red line represents that besides the treatment by an external input of IL-2 into the system, there is also the treatment by an external source of effector cells, i.e., $s_{2}=0.5$ ). (b) Drawing of partial enlargement of 5(a).

cells and the treatment by an external input of IL-2 into the system, respectively. For better visualization of the effects of two types of immunotherapy, we consider the rate of tumor extinction under two cases: $s_{2}$ with different $s_{1}$, or $s_{1}=3$ with different $s_{2}$. Figure 4 implies the former case, Figures 5 and 6 imply the latter case. The results show

(1) Tumor treatment by an external source of effector cells, i.e., $s_{2}=0$ with different $s_{1}$. Figure 4 shows that the higher the value of $s_{1}$, the asymptotic rate of convergence of $v$ or the rate of tumor extinction will be larger; however, the variations are not obvious when $s_{1}$ reaches a critical value.
(2) Tumor treatment by an external source of effector cells without or with an external input of IL-2 into the system, i.e., $s_{1}=3, s_{2}=0$ or $s_{1}=3, s_{2}=0.5$. Figure 5 shows that the introduction of new immunotherapy methods has accelerated the asymptotic rate of convergence of $v$ or the rate of tumor extinction.

(3) Tumor treatment by an external source of effector cells and an external input of IL-2 into the system, i.e., $s_{1}=3$ with different $s_{2}$. Figure 6 shows that with the same value of $s_{1}$ and higher value of $s_{2}$, the asymptotic rate of convergence of $v$ or the rate of tumor extinction will be larger; however, the 


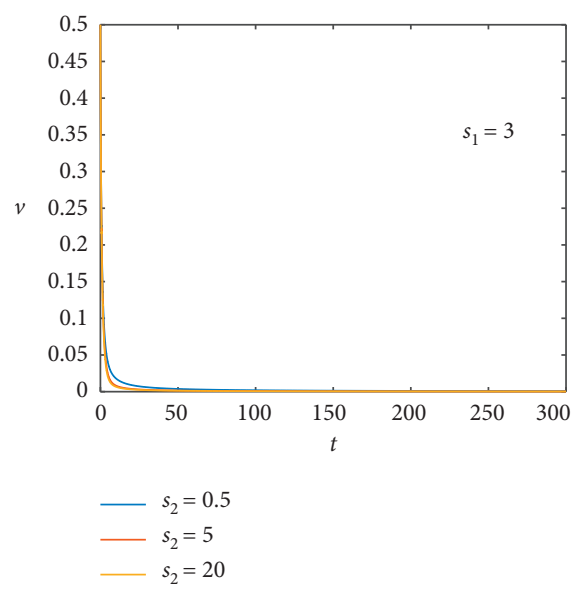

(a)

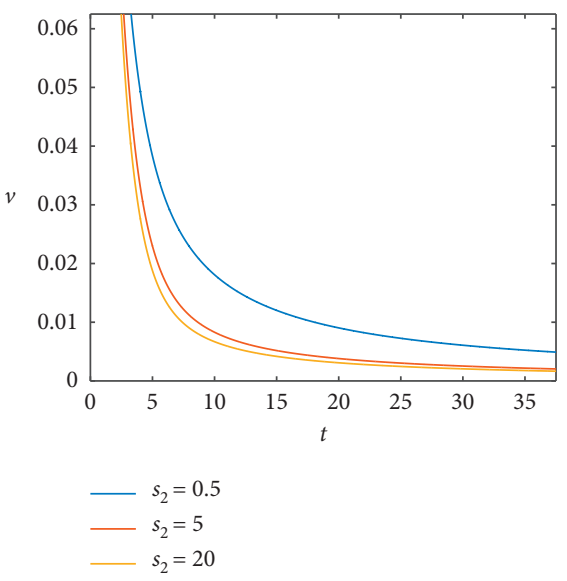

(b)

Figure 6: Time series of the immune system cells $v$ in the fractional-order model (4) with $c=0.9, \mu_{2}=1, p_{1}=0.5, s_{1}=3, b=3, a=1$, $g=2.5, p_{2}=1, \mu_{3}=1, \alpha=0.9$, and different treatment by an external source of effector cells $s_{2}$ : (a) original drawing and (b) drawing of partial enlargement of $6(\mathrm{a})$.

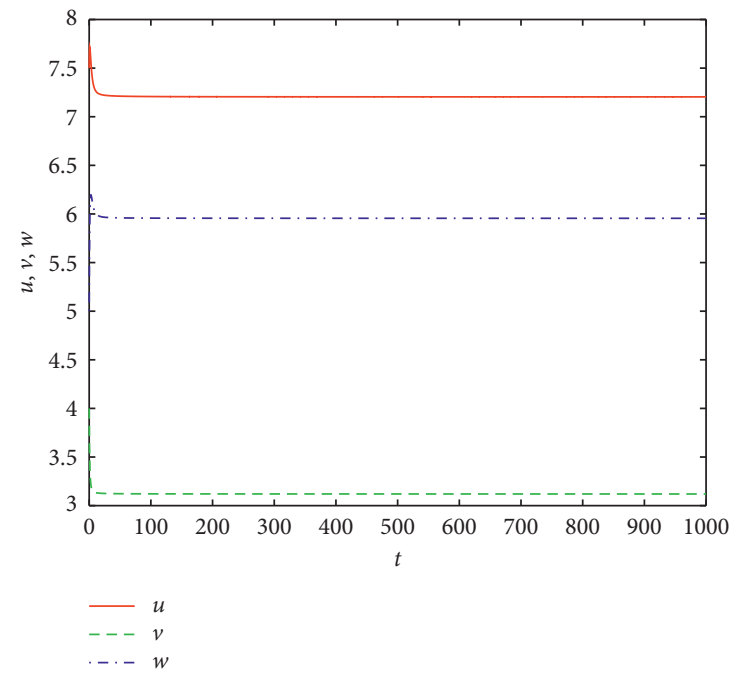

Figure 7: Time series of the fractional-order model (4) with $c=1, \mu_{2}=1, p_{1}=0.5, s_{1}=1, b=0.3, a=0.05, g=2.5, p_{2}=1, \mu_{3}=1$, $s_{2}=0.5$, and $\alpha=0.9$.

variations are not obvious when $s_{2}$ reaches a critical value.

In other words, the desired best effects can be achieved by combining the two types of immunotherapy.

Second, we choose the following set of parameters:

$$
\begin{aligned}
c & =1, \\
\mu_{2} & =1, \\
p_{1} & =0.5, \\
s_{1} & =1, \\
s_{2} & =0.5,
\end{aligned}
$$

and consider the asymptotic stability of the unique interior equilibrium $E_{*}$. This yields that under some conditions, combination therapy (ACI plus IL-2) can achieve satisfactory and stable tumor control; however, the tumor is incurable.

Figures 7 and 8 indicate that the unique interior equilibrium

$$
E_{*}=(7.2036,3.1197,5.9551) \text { or }(2.5191,0.7481,1.5781) \text {, }
$$

is asymptotically stable when $b=0.3, a=0.05, g=2.5, p_{2}=1$, $\mu_{3}=1$, and $\alpha=0.9 \quad$ or $\quad b=0.3, a=1, g=2.5, p_{2}=1, \mu_{3}=1$, and $\alpha=0.6$, respectively, concurring with the results of Theorem 5 (1) and (2).

Figure 9 indicates that all trajectories with different positive initial conditions converge to the unique interior equilibrium $E_{*}=(0.8463,0.5880,0.3283)$ when $b=1.5$, $a=0.5, g=3, p_{2}=0.5, \mu_{3}=2$, and $\alpha=0.9$, which indicates that $E_{*}$ is globally asymptotically stable, concurring 


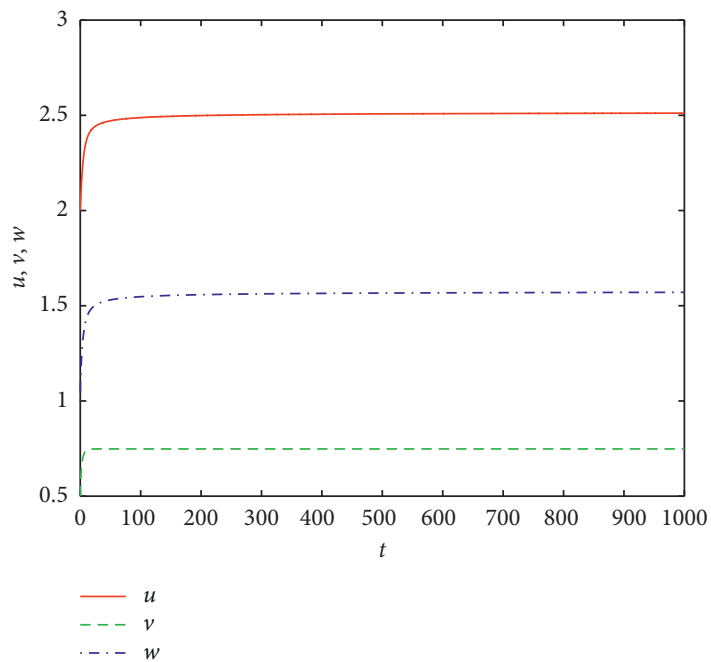

Figure 8: Time series of the fractional-order model (4) with $c=1, \mu_{2}=1, p_{1}=0.5, s_{1}=1, b=0.3, a=1, g=2.5, p_{2}=1, \mu_{3}=1, s_{2}=0.5$, and $\alpha=0.6$.
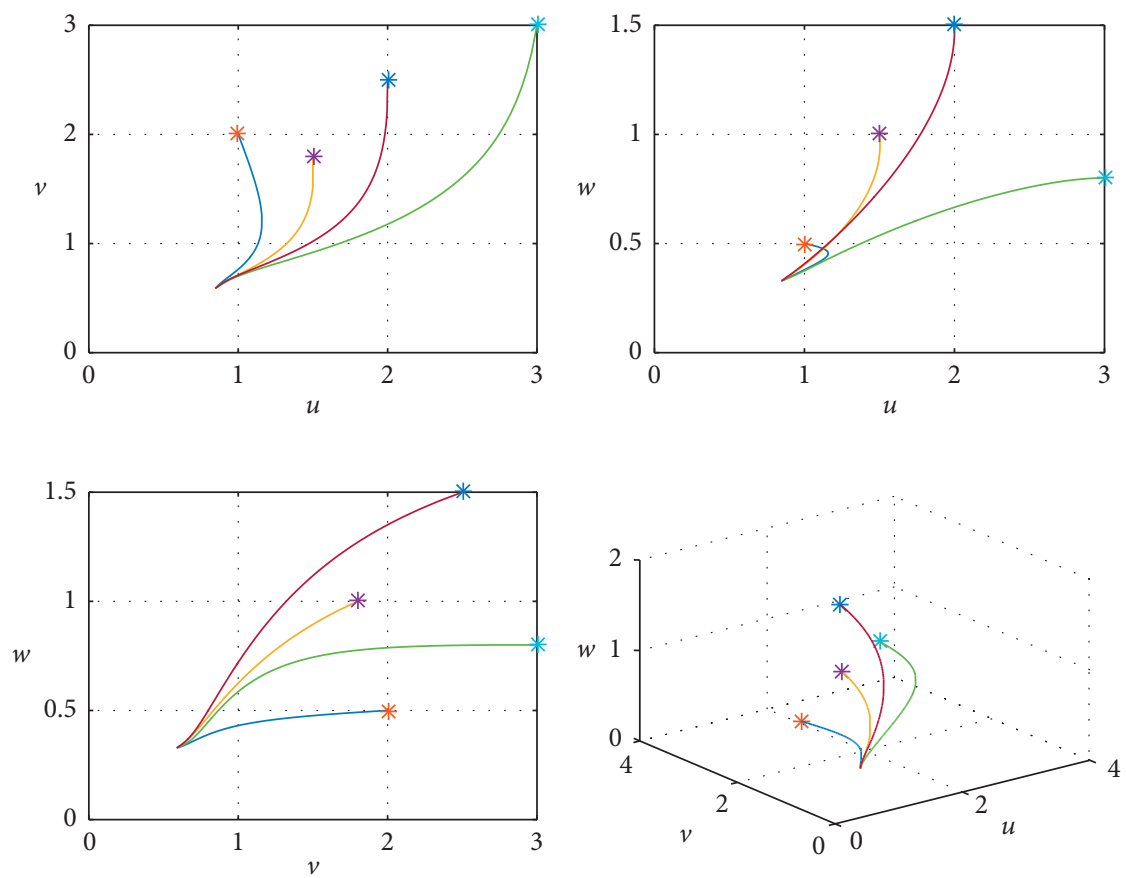

Figure 9: Phase diagram of the fractional-order model (4) with $c=1, \mu_{2}=2, p_{1}=0.5, s_{1}=1, b=1.5, a=0.5, g=$ $3, p_{2}=0.5, \mu_{3}=2, s_{2}=0.5, \alpha=0.9$, and different initial conditions $\left(u_{0}, v_{0}, w_{0}\right)$.

with the results of Theorem 6 . This situation means that the tumor will exist indefinitely, which will be incurable in medicine.

\section{Concluding Remarks}

In this paper a fractional-order tumor-immune interaction model with immunotherapy is discussed. The existence, uniqueness, and nonnegativity of the solutions are proved. The local and global asymptotic stability of some equilibrium points are investigated. Unfortunately, by the fractional calculation, we cannot obtain the boundedness of solutions to the fractional-order tumor-immune model (4) with $\alpha \in(0,1)$.

In addition, numerical simulations are conducted to illustrate the analytical results. This yields that under some conditions, the tumor can be cured thoroughly, by the therapy (ACI or ACI plus IL-2); under some other conditions, combination therapy (ACI plus IL-2) can achieve satisfactory and stable tumor control; however, the tumor is incurable. The results indicate that the sufficiently large order $\alpha$ of the Caputo fractional derivative has a stabilization effect, and it may help to control the tumor extinction, in the tumor-immune model with immunotherapy. 


\section{Data Availability}

The data used to support the findings of this study are available from the corresponding author upon request

\section{Conflicts of Interest}

The authors declare that they have no conflicts of interest.

\section{Acknowledgments}

The work was supported by National Natural Science Foundation of China (no. 81571731), Key Research and Development Program of Shaanxi (no. 2018SF-161), Natural Science Basic Research Plan in Shaanxi Province of China (no. 2020JM-569), and Scientific Research Program Funded by Shaanxi Provincial Education Department (no. 19JK0792)

\section{References}

[1] J. Marx, "How cancer cells spread in the body," Science, vol. 244, no. 4901, pp. 147-148, 1989.

[2] G. P. Dunn, A. T. Bruce, H. Ikeda, L. J. Old, and R. D. Schreiber, "Cancer immunoediting: from immunosurveillance to tumor escape," Nature Immunology, vol. 3, no. 11, pp. 991-998, 2002.

[3] N. P. Restifo, M. E. Dudley, and S. A. Rosenberg, "Adoptive immunotherapy for cancer: harnessing the T cell response," Nature Reviews Immunology, vol. 12, no. 4, pp. 269-281, 2012.

[4] W. H. West, K. W. Tauer, J. R. Yannelli et al., "Constantinfusion recombinant interleukin-2 in adoptive immunotherapy of advanced cancer," New England Journal of Medicine, vol. 316, no. 15, pp. 898-905, 1987.

[5] F. K. Nani and M. N. Oğuztöreli, "Modelling and simulation of Rosenberg-type adoptive cellular immunotherapy," Mathematical Medicine and Biology, vol. 11, no. 2, pp. 107147, 1994.

[6] D. Kirschner and J. C. Panetta, "Modeling immunotherapy of the tumor-immune interaction," Journal of Mathematical Biology, vol. 37, no. 3, pp. 235-252, 1998.

[7] N. Heymans and I. Podlubny, "Physical interpretation of initial conditions for fractional differential equations with Riemann-Liouville fractional derivatives," Rheologica Acta, vol. 45, no. 5, pp. 765-771, 2006.

[8] H.-L. Li, L. Zhang, C. Hu, Y.-L. Jiang, and Z. Teng, "Dynamical analysis of a fractional-order predator-prey model incorporating a prey refuge," Journal of Applied Mathematics and Computing, vol. 54, no. 1-2, pp. 435-449, 2017.

[9] I. Petráš, Fractional-order Nonlinear Systems: Modeling, Analysis and Simulation, Springer Science \& Business Media, Berlin, Germany, 2011.

[10] M. A. Khan, M. Ismail, S. Ullah, and M. Farhan, "Fractional order sir model with generalized incidence rate," AIMS Mathematics, vol. 5, no. 3, pp. 1856-1880, 2020.

[11] M. A. Khan, A. Khan, A. Elsonbaty, and A. A. Elsadany, "Modeling and simulation results of a fractional dengue model," The European Physical Journal Plus, vol. 134, no. 8, p. 379, 2019.

[12] S. Ullah, M. A. Khan, M. Farooq, T. Gul, and F. Hussain, "A fractional order HBV model with hospitalization," Discrete \& Continuous Dynamical Systems-S, vol. 13, no. 3, pp. 957-974, 2019.
[13] M. A. Khan, S. Ullah, and M. Farhan, "The dynamics of zika virus with caputo fractional derivative," AIMS Mathematics, vol. 4, no. 1, pp. 134-146, 2019.

[14] A. A. A. Kilbas, H. M. Srivastava, and J. J. Trujillo, Theory and Applications of Fractional Differential Equations, Vol. 204, Elsevier Science Limited, Amsterdam, Netherlands, 2006.

[15] Y. Li, Y. Chen, and I. Podlubny, "Stability of fractional-order nonlinear dynamic systems: Lyapunov direct method and generalized Mittag-Leffler stability," Computers \& Mathematics with Applications, vol. 59, no. 5, pp. 1810-1821, 2010.

[16] B. Bandyopadhyay and S. Kamal, Stabilization and Control of Fractional Order Systems: A Sliding Mode Approach, Vol. 317, Springer, Berlin, Germany, 2015.

[17] S. K. Choi, B. Kang, and N. Koo, "Stability for caputo fractional differential systems," Abstract and Applied Analysis, vol. 2014, Article ID 631419, 6 pages, 2014.

[18] W. Ko and I. Ahn, "Stationary patterns and stability in a tumor-immune interaction model with immunotherapy," Journal of Mathematical Analysis and Applications, vol. 383, no. 2, pp. 307-329, 2011.

[19] E. Ahmed, A. M. A. El-Sayed, and H. A. A. El-Saka, "On some Routh-Hurwitz conditions for fractional order differential equations and their applications in Lorenz, Rössler, Chua and Chen systems," Physics Letters A, vol. 358, no. 1, pp. 1-4, 2006.

[20] C. Vargas-De-León, "Volterra-type Lyapunov functions for fractional-order epidemic systems," Communications in Nonlinear Science and Numerical Simulation, vol. 24, no. 1-3, pp. 75-85, 2015.

[21] J. I. Diaz, T. Pierantozzi, and L. Vazquez, "Finite time extinction for nonlinear fractional evolution equations and related properties," Electronic Journal of Differential Equations, vol. 2016, no. 239, pp. 1-13, 2016.

[22] A. Alsaedi, B. Ahmad, and M. Kirane, "A survey of useful inequalities in fractional calculus," Fractional Calculus and Applied Analysis, vol. 20, no. 3, pp. 574-594, 2017.

[23] K. Diethelm, N. J. Ford, and A. D. Freed, "A predictor-corrector approach for the numerical solution of fractional differential equations," Nonlinear Dynamics, vol. 29, no. 1-4, pp. 3-22, 2002.

[24] R. Garrappa, "Trapezoidal methods for fractional differential equations: theoretical and computational aspects," Mathematics and Computers in Simulation, vol. 110, pp. 96-112, 2015. 